\title{
Association of Carotid Artery Endothelial Signal Intensity Gradient with Unilateral Large Artery Ischemic Stroke
}

\author{
Chan-Hyuk Lee $^{a}$ Sang Hyuk Lee ${ }^{b}$ Young I. Choc Seul-Ki Jeong ${ }^{d}$ \\ aDepartment of Neurology, Jeonbuk National University Hospital, Research Institute of Clinical Medicine, \\ Biomedical Research Institute of Jeonbuk National University Hospital, Jeonbuk National University, Jeonju, \\ Republic of Korea; ${ }^{b}$ Equipment Qualification Center for Nuclear Power Plants, Korea Institute of Machinery and \\ Materials, Daejeon, Republic of Korea; 'Department of Mechanical Engineering and Mechanics, Drexel University, \\ Philadelphia, PA, USA; dVisual Intelligence Laboratory, Seul-Ki Jeong Neurology Clinic, Seoul, Republic of Korea
}

\section{Keywords}

Carotid artery $\cdot$ Endothelial shear stress · Ischemic stroke · Large artery atherosclerosis · Signal intensity gradient

\begin{abstract}
Background: Common carotid artery (CCA) and internal carotid artery (ICA) are aligned linearly, but their hemodynamic role in ischemic stroke has not been studied in depth. $\mathbf{O b}$ jectives: We aimed to investigate whether CCA and ICA endothelial shear stress (ESS) could be associated with the ischemic stroke of large artery atherosclerosis (LAA). Methods: We enrolled consecutive patients with unilateral ischemic stroke of LAA and healthy controls aged $>60$ years in the stroke center of Jeonbuk National University Hospital. All patients and controls were examined with carotid artery time-of-flight magnetic resonance angiography, and their endothelial signal intensity gradients (SIGs) were determined, as a measure of ESS. The effect of right or left unilateral stroke on the association between carotid artery endothelial SIG and ischemic stroke of LAA was assessed. Results: In total, the results from 132 patients with ischemic stroke of LAA and 121 controls were analyzed. ICA endothelial SIG showed significant and independent associations with the same-sided unilateral ischemic stroke of LAA, even after ad-
\end{abstract}

justing for the potential confounders including carotid stenosis, whereas CCA endothelial SIG showed a significant association with the presence of the ischemic stroke of LAA. Conclusion: Although CCA and ICA are located with continuity, the hemodynamics and their roles in large artery ischemic stroke should be considered separately. Further studies are needed to delineate the pathophysiologic roles of ESS in CCA and ICA for large artery ischemic stroke.

두 2021 S. Karger AG, Base

\section{Introduction}

Large artery ischemic stroke is a huge global burden, causing moderate to severe disability and even fatality [1]. The common causes of large artery ischemic stroke include intracranial artery stenosis (ICAS) or artery-to-artery embolization from proximal area [2]. Therefore, extracranial carotid bifurcation might have a pivotal role in escalating the risks of unilateral stroke according to the presence of pathologic lesions [3]. For the ischemic stroke of large artery atherosclerosis (LAA) according to the Trial of Org 10,172 in Acute Stroke Treatment (TOAST) classification, an extracranial carotid artery stenosis of $>50 \%$ is a diagnostic component [4].

$\begin{aligned} & \text { karger@karger.com } \\ & \text { www.karger.com/ced }\end{aligned}$
Karger ${ }^{\prime /}$


Endothelial shear stress (ESS) is a biomechanical tangential force due to the blood flow applied to the walls of blood vessels. It is essential to maintain a normal range of ESS in order to keep artery healthy and atheroprotective [5]. Low ESS is known to promote atherosclerosis by causing functional abnormalities of vascular endothelial cells [6]. It was reported that both coronary [7] and basilar artery [8] ESS were associated with the progression and transformation of atherosclerotic plaques. Common carotid artery (CCA) ESS was associated with the white matter hyperintensities and cognitive function [9]. However, CCA and ICA have not been studied separately for a relationship with ischemic stroke, even though CCA and ICA were known to have different hemodynamics features [10].

In the previous study, carotid artery endothelial signal intensity gradient (SIG) from time-of-flight magnetic resonance angiography (TOF-MRA) was reported to represent the relative ESS, showing strong positive correlations with wall shear stress from computational fluid dynamics [11]. TOF-MRA, which is based on the physiologic feature of flow enhancement in the construction of vascular imaging, is one of the most commonly used angiographies $[12,13]$. In the terminal internal carotid artery (ICA) that appears normal, an increased spatial variability of the SIG was associated with the progression of moyamoya disease [14].

In the present study, we aimed to investigate whether the CCA and ICA endothelial SIG values were associated with the ischemic stroke of LAA, and whether the association was affected by the right or left stroke-sidedness. To accomplish the goal, the endothelial SIG was measured and analyzed from the CCA and ICAs separately in the patients with ischemic stroke of LAA and control subjects.

\section{Materials and Methods}

\section{Study Population}

This is a case-control study. Consecutive patients referred to the Stroke Centre and control participants who visited health checkup program at Jeonbuk National University Hospital from June 2012 to May 2013 were included in the study. The examinees' medical information, including age, sex, smoking, and alcohol consumption (current) was recorded. If a patient had a history of past smoking, the patient was defined as having a history of smoking. Hypertension was defined as having been diagnosed with hypertension in the past, taking current medication for hypertension, or blood pressure equal to or $>140 / 90 \mathrm{~mm} \mathrm{Hg}$ under stable conditions. Type 2 diabetes mellitus was defined as a history of previous diabetes, current medication for diabetes, or a fasting blood sugar measurement equal to or $>126 \mathrm{mg} / \mathrm{dL}$ on more than 2 occasions. Serum lipid profile and albumin level were tested using standard methods. We obtained informed consent from the participants.
This study was conducted following approval from the ethics review committee (CUH2013-03-007-001). Also, the committee confirmed that our study was performed in accordance with relevant guidelines and regulations (Human Research Protection Program, version 2.0, Jeonbuk National University Hospital Medical Ethics Review Committee, Republic of Korea).

\section{Assessment for Ischemic Stroke and Selection of Patient and} Control Group

Ischemic stroke was defined by acute focal neurological deficits lasting $>24 \mathrm{~h}$, and cerebral ischemia diagnosed on brain imaging such as computed tomography and/or magnetic resonance imaging. Ischemic stroke is categorized originally into 5 groups: LAA, cardioembolism, small vessel occlusion (SVO) or lacunar infarction, stroke of other determined etiology, and stroke of undetermined etiology based upon the diagnostic criteria of the Trial of Org 10172 in Acute Stroke Treatment (TOAST) study [4]. In the present study, only patients with LAA subtype involving anterior circulation were included and proceeded for the examination of TOF-MRA of carotid artery. The control group was selected among those who participated in the regular health screening and carotid TOF-MRA in the same hospital. The control group was defined as the participants with no previous history of vascular events such as myocardial infarction, any stroke, and peripheral arterial disease.

\section{Exclusion Criteria}

The patients and controls with the following characteristics were excluded: (1) patients diagnosed with at least 1 ICA occlusion (right or left), (2) patients performed for carotid artery revascularization including endarterectomy and/or stenting, (3) patients with ischemic stroke other than LAA subtype involving anterior circulation, (4) patients who were examined by contrast-enhanced carotid MRA, (5) controls with abnormal hepatic (aspartate aminotransferase, alanine aminotransferase, alkaline phosphatase, bilirubin, and albumin), renal (Cr, and glomerular filtration rate), or hematologic (complete blood count, thrombin time, prothrombin time, and activated partial thromboplastin time) laboratory findings, and (6) participants who aged $<60$ years.

\section{Three-Dimensional TOF-MRA Examinations and Carotid Ultrasonography}

Carotid artery three-dimensional TOF-MRA was performed at Jeonbuk National University Hospital. The imaging parameters for the three-dimensional TOF-MRA scan were repetition time (TR)/ echo time $(\mathrm{TE})=23 / 3.5 \mathrm{~ms}$; flip angle $=20.0^{\circ}$; field of view $($ FOV $)=200 \times 200 \mathrm{~mm}$; acquired matrix size $=488 \times 249$; sensitivity encoding factor $=2.5$; slice thickness $=1.2 \mathrm{~mm}$; total slice number $=180$; and the number of average $=1$. The TOF-MRA scan time (Achieva 3.0T, Philips) was $4 \mathrm{~min}$. Both CCA and proximal portion of internal carotid artery (ICA) were included for scanning.

For a proper measurement of carotid stenosis or occlusion, duplex ultrasonography was performed with electrocardiogram triggering and a high-frequency $5-12 \mathrm{MHz}$ (12L5) linear transducer (Terason $\mathrm{t} 3000$, Teratech Corporation, Burlington, MA, USA) by a certified neurosonographer, as reported previously [15]. The ultrasonographic examination was done within 1 week after stroke events or at the same day with TOF-MRA during the health checkup. Carotid duplex ultrasonography was performed along the CCA and ICA at the both sides. The degree of ICAS at both grayscale and Doppler ultrasonography was stratified according to 


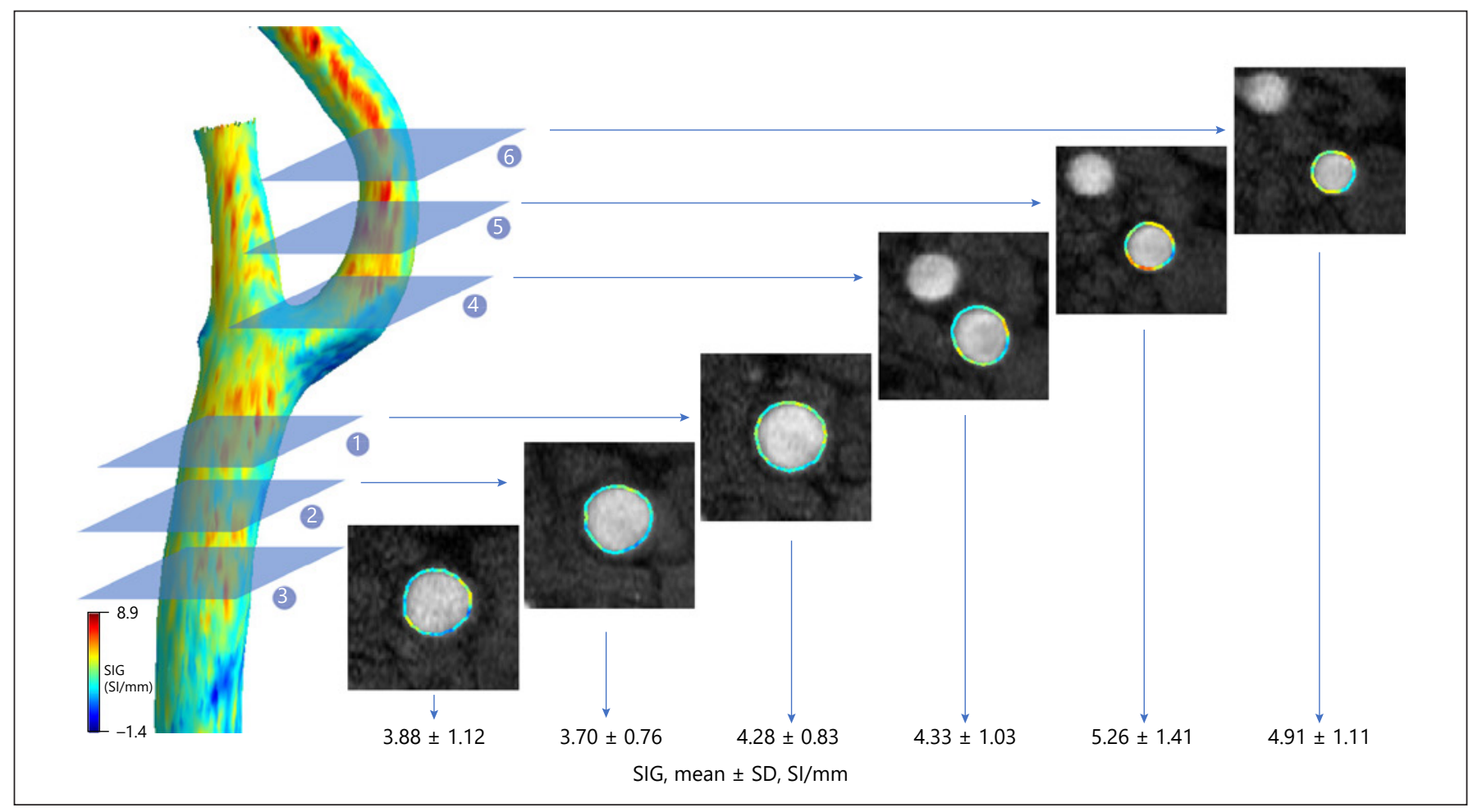

Fig. 1. A schematic layout for a measurement of endothelial SIG from TOF-MRA. For the CCA, the junction of the carotid bulb and the distal CCA was set as the base (number 1), and with a 6-mm interval, 2 more proximal levels were sequentially set as number 2 and 3. For the ICA, the dividing portion into ICA and ECA was set

peak systolic/end-diastolic velocity and the presence of plaque, as previously recommended [16]. In brief, carotid stenosis was defined as $>50 \%$ stenosis with ICA peak systolic velocity $>125 \mathrm{~cm} / \mathrm{s}$, while plaque is visible or luminal narrowing is seen.

\section{Measurement of Carotid Artery Endothelial SIG}

Carotid artery segmentation and endothelial SIG measurement were performed with a semi-automated software (VINT, mediimage, Inc.), as reported previously [11]. In brief, the signal intensities at the iso-point $\left(\Phi_{\mathrm{a}}\right.$; signal intensity at position $A\left[\mathrm{X}_{\mathrm{a}}\right]$ along the arterial contour line $)$ and at the inner point $\left(\Phi_{\mathrm{b}}\right.$; signal intensity at position $B\left[\mathrm{X}_{\mathrm{b}}\right]$ ) were calculated by using a trilinear interpolation algorithm based on the positions and signal intensities in the 8 neighboring voxels. The signal intensities of TOF-MRA were normalized to eliminate the offset and scale effects across the MRA datasets of participants. For each iso-point (position A), the SIG was calculated from the difference in signal intensities between points $\mathrm{A}$ and $\mathrm{B}$ as follows:

$$
\text { SIG, SI/mm }=\left(\Phi_{\mathrm{b}}-\Phi_{\mathrm{a}}\right) /\left|\mathrm{X}_{\mathrm{b}}-\mathrm{X}_{\mathrm{a}}\right|
$$

The endothelial SIG values were obtained from 3 levels at both CCAs and ICAs, as shown in Figure 1. For the CCA, the diverging portion of the distal CCA was set as the base (number 1), and 2 more proximal levels were set with $6-\mathrm{mm}$ intervals as number 2 and 3 for the measurements of endothelial SIG. For the ICA, the as the base (number 4), and with a 6-mm interval, 2 more distal levels were sequentially set as number 5 and 6 . SIG, signal intensity gradient; CCA, common carotid artery; ICA, interval carotid artery; ECA, external carotid artery; TOF-MRA, time-of-flight magnetic resonance angiography.

dividing portion was set as the base (number 4), and 2 more distal levels were set with the same 6 - $\mathrm{mm}$ intervals as number 5 and 6 . The mean values of endothelial SIG from the 3 CCA and 3 ICA levels were calculated and used in the present analysis.

\section{Statistical Analysis}

Descriptive data for the clinical characteristics and laboratory findings of the 2 groups were expressed as a mean \pm standard deviation or percentage as appropriate. Kolmogorov-Smirnov test was performed for distributional adequacy. Independent $t$-test was used to assess differences in the continuous variables and a $\chi^{2}$ test for the categorical variables. Paired $t$-test was used to assess differences of right versus left carotid artery endothelial SIG values. Multivariate logistic regression analysis was performed to show independent associations between carotid artery endothelial SIG and ischemic stroke of LAA. Covariates were selected based on a biological plausibility and clinical relevance for ischemic stroke, including age, sex, smoking, hypertension, type 2 diabetes, high-density lipoprotein cholesterol, albumin, and ICAS in the final model. In addition, the analysis was performed separately according to the stroke-sidedness because of the potential modification effects. Interaction terms between the stroke-sidedness and carotid artery wall SIG values were created, and their significance was assessed by likelihood ratio tests. $p$ value $<0.05$ was considered significant. All statistical analyses were conducted using SPSS 23.0 for Windows (IBM Corp., Armonk, NY, USA). 


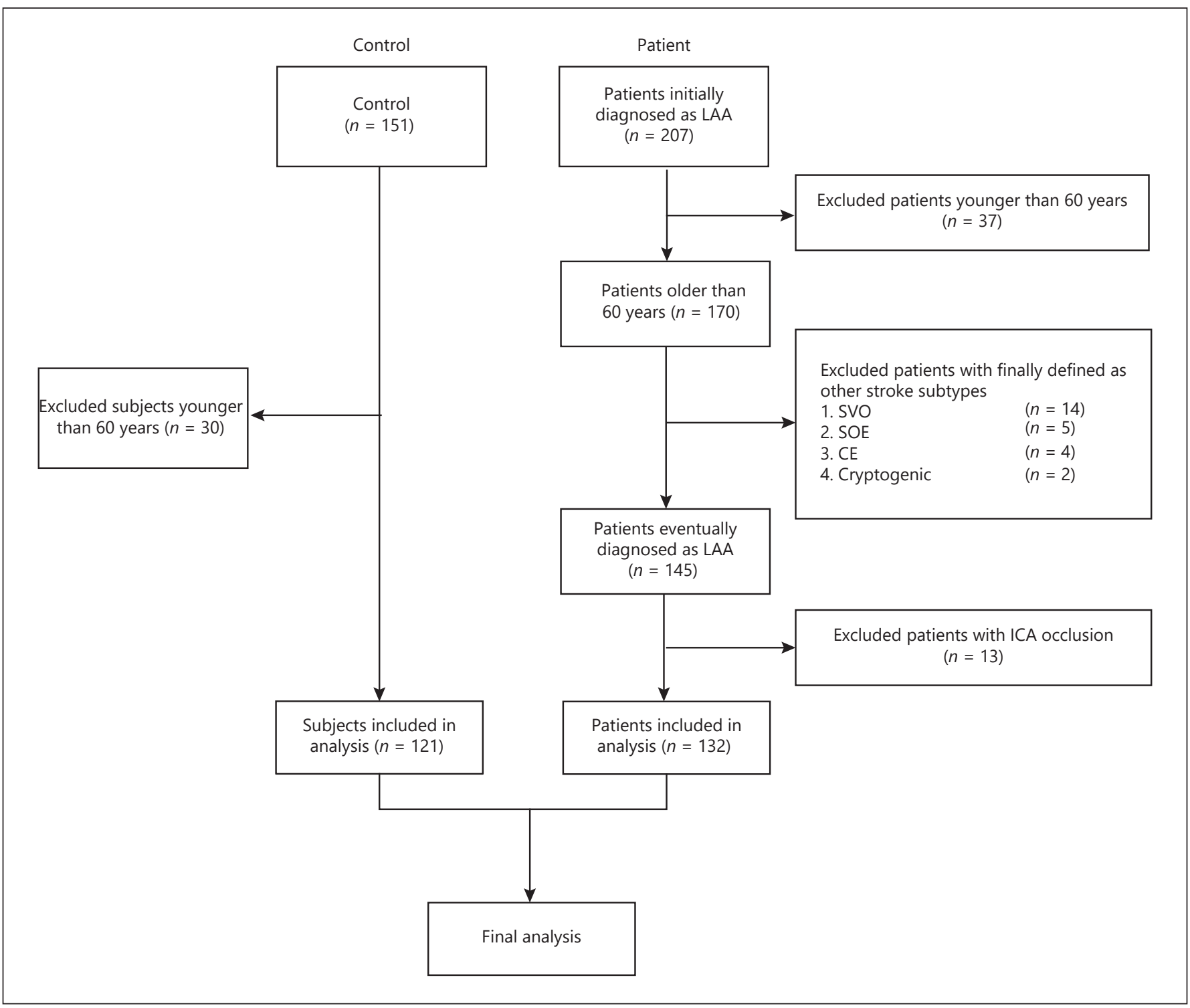

Fig. 2. A screening flow chart for patient and control group. LAA, large artery atherosclerosis; SVO, small vessel occlusion; SOE, stroke of other determined etiology; CE, cardioembolism; ICA, internal carotid artery.

\section{Results}

In total, 207 patients with ischemic stroke who were initially diagnosed as LAA and 151 controls were examined with carotid TOF-MRA from June 2012 to May 2013. Among the patients, $37(17.9 \%)$ were $<60$ years of age, $25(12.1 \%)$ were finally defined as other stroke subtypes (14 SVO, 5 stroke of other determined etiology, 4 cardioembolism, and 2 cryptogenic in stroke of undetermined etiology), and 13 (6.3\%) were diagnosed as ICA occlusion (8 right-sided and 5 left-sided), all of whom were excluded in the final analysis. Among the controls, $30(19.9 \%)$ were excluded from the study because of exclusion criteria for age. In total, 132 patients with ischemic stroke of LAA type and 121 controls were analyzed (Fig. 2).

Demographic and hemodynamic factors are shown in Table 1. Patients with ischemic stroke of LAA were older and showed lower high-density lipoprotein and serum albumin concentration than control group. All the endothelial SIG values in the both CCA and ICAs were lower in the patient group. 
Table 1. Demographics

\begin{tabular}{|c|c|c|c|}
\hline Variables & Controls & Patients & $p$ value* \\
\hline$N$ & 121 & 132 & \\
\hline Age, years & $71.7 \pm 6.4$ & $74.3 \pm 7.3$ & 0.003 \\
\hline Female, $\%$ & 47.9 & 47.4 & 0.928 \\
\hline Hypertension, \% & 62.5 & 68.0 & 0.366 \\
\hline Type 2 diabetes, $\%$ & 33.3 & 39.2 & 0.340 \\
\hline Smoking, \% & 13.6 & 16.0 & 0.592 \\
\hline LDL cholesterol, mg/dL & $93.1 \pm 30.3$ & $93.0 \pm 28.1$ & 0.984 \\
\hline HDL cholesterol, mg/dL & $45.3 \pm 13.9$ & $39.0 \pm 9.4$ & $<0.001$ \\
\hline Albumin, g/dL & $4.3 \pm 0.4$ & $4.1 \pm 0.4$ & 0.001 \\
\hline Right ICA, stenosis, ${ }^{\dagger} \%$ & 13.2 & 18.8 & 0.228 \\
\hline Left ICA, stenosis, ${ }^{\dagger} \%$ & 17.4 & 13.8 & 0.307 \\
\hline Right or left ICAS, ${ }^{\dagger} \%$ & 20.7 & 26.3 & 0.289 \\
\hline \multicolumn{4}{|c|}{ Carotid artery endothelial SIG, SI/mm } \\
\hline Right CCA & $3.2 \pm 0.9$ & $2.9 \pm 0.6$ & 0.003 \\
\hline Right ICA & $3.5 \pm 0.9$ & $3.2 \pm 0.6$ & 0.031 \\
\hline Left CCA & $3.1 \pm 0.8$ & $2.9 \pm 0.6$ & 0.006 \\
\hline Left ICA & $3.4 \pm 1.0$ & $3.0 \pm 0.7$ & $<0.001$ \\
\hline
\end{tabular}

Values represented as mean \pm standard deviation (SD). LDL, low-density lipoprotein; HDL, high-density lipoprotein; ICA, internal carotid artery; ICAS, internal carotid artery stenosis; CCA, common carotid artery; SIG, signal intensity gradient; TOF-MRA, time-of-flight magnetic resonance angiography. * $p$ values as determined by independent $t$-test or $\chi^{2}$ test as appropriate. ${ }^{\dagger}$ Carotid stenosis was defined as more than $50 \%$ by ultrasonographic examination, showing peak systolic velocity $>125 \mathrm{~cm} / \mathrm{s}$, while plaque is visible or luminal narrowing seen [16].

Table 2. Right versus left carotid artery endothelial SIG

\begin{tabular}{|c|c|c|c|}
\hline & \multicolumn{3}{|c|}{ Artery wall SIG, SI/mm } \\
\hline & right & left & $p$ value* \\
\hline \multicolumn{4}{|l|}{ Control, $N=121$} \\
\hline CCA & $3.2 \pm 0.9$ & $3.1 \pm 0.8$ & 0.822 \\
\hline Internal carotid artery & $3.5 \pm 0.9$ & $3.4 \pm 1.0$ & 0.912 \\
\hline \multicolumn{4}{|c|}{ Patients with right-sided ischemic stroke of LAA, $N=64$} \\
\hline CCA & $2.8 \pm 0.7$ & $3.0 \pm 0.6$ & 0.035 \\
\hline Internal carotid artery & $3.0 \pm 0.8$ & $3.3 \pm 0.8$ & 0.047 \\
\hline \multicolumn{4}{|c|}{ Patients with left-sided ischemic stroke of LAA, $N=68$} \\
\hline CCA & $3.2 \pm 0.6$ & $2.9 \pm 0.6$ & 0.039 \\
\hline Internal carotid artery & $3.3 \pm 0.6$ & $2.8 \pm 0.7$ & $<0.001$ \\
\hline
\end{tabular}

Values represented as mean \pm SD. SIG, signal intensity gradient; CCA, common carotid artery; LAA, large artery atherosclerosis. ${ }^{*} p$ values as determined by paired $t$ test.

In the control group, there was no side-to-side difference of carotid endothelial SIG (right vs. left CCA SIG, $\mathrm{SI} / \mathrm{mm}, 3.2 \pm 0.9$ vs. $3.1 \pm 0.8$; right vs. left ICA SIG $3.5 \pm$ 0.9 vs. $3.4 \pm 1.0, p>0.5$ ), as shown in Table 2 . On the contrary, there were significant side-to-side differences of carotid endothelial SIG in the stroke patients according to the stroke-sidedness, as shown in Figure 3. In the patients with the right ischemic stroke of LAA, carotid endothe- lial SIGs were significantly lower in the right than the left (right vs. left CCA SIG, $2.8 \pm 0.7$ vs. $3.0 \pm 0.6, p=0.035$; right vs. left ICA SIG $3.0 \pm 0.8$ vs. $3.3 \pm 0.8, p=0.047$, respectively). In the patients with left ischemic stroke of LAA, left CCA and ICA showed significantly lower SIG values than the right, as shown in Table 2 (see online suppl. Table 1; see www.karger.com/doi/10.1159/000514141 for all online suppl. material). 


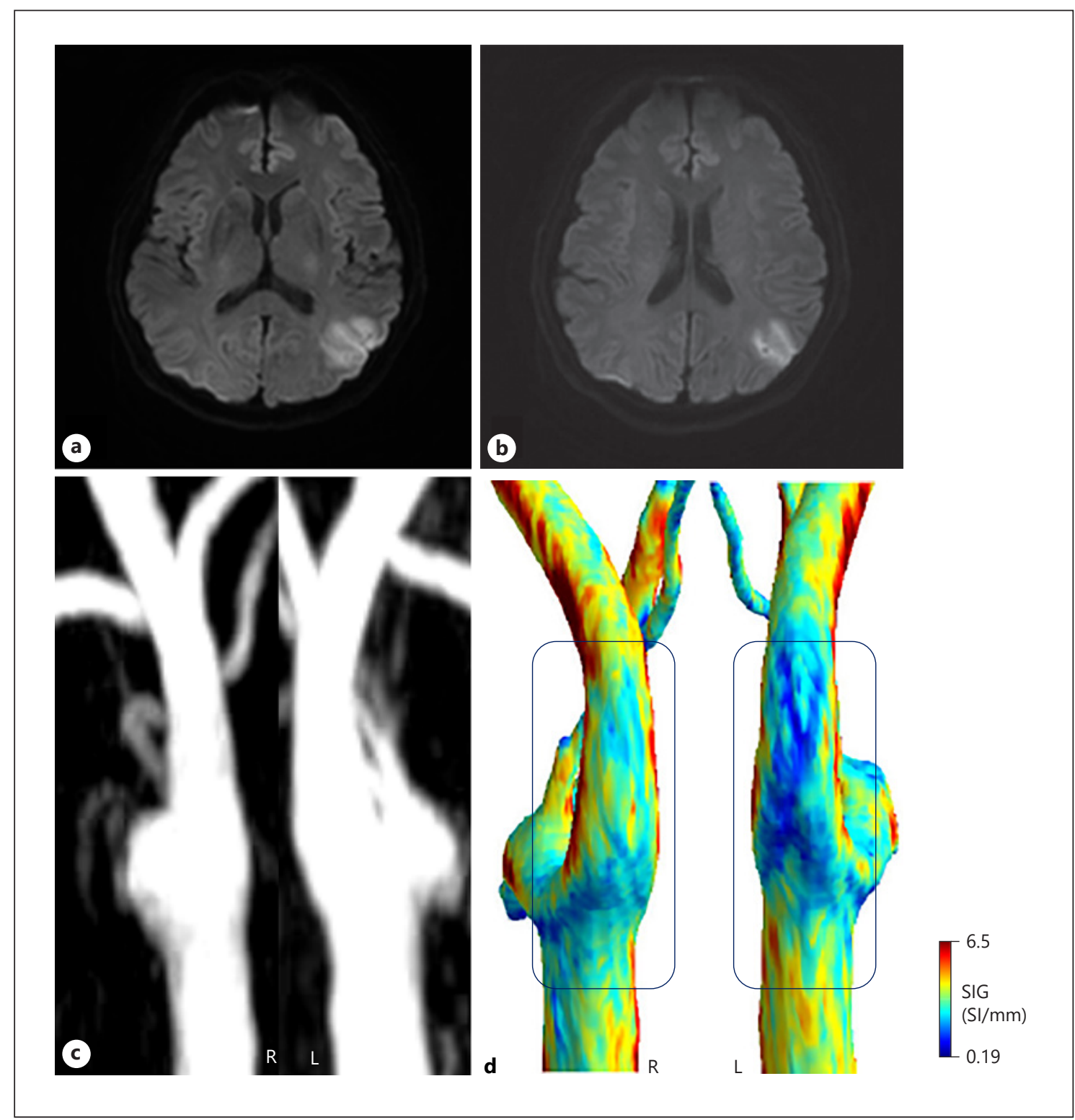

Fig. 3. A representative case with a left hemispheric ischemic lesion. A 52-year-old male reported sensory aphasia with transient right-sided hemiparesis. a, b Brain DWI shows high signal intensity lesion in the branch artery of left middle cerebral artery. $\mathbf{c}$ Both carotid arteries have no significant stenosis in carotid TOF-MRA. d Lesion-sided left carotid artery (right square) showed lower endothelial SIG than right carotid artery (left square). SIG, signal intensity gradient; DWI, diffusion weighted image; TOF-MRA, time-of-flight magnetic resonance angiography.

right ICA endothelial SIG showed independent associations (OR, 0.50 and 95\% CI, 0.29-0.86), but the left ICA did not. For the left-sided ischemic stroke, the left ICA endothelial SIG showed independent association (OR, 0.43 ; 95\% CIs, 0.20-0.94), but the right ICA did not. Interaction terms between stroke-sidedness and both ICA endothelial SIG values were significant ( $p=0.008$ for left ICA and $p=0.014$ for right ICA, respectively). 
Table 3. Multivariate associations between carotid artery endothelial SIG and ischemic stroke of LAA

\begin{tabular}{|c|c|c|c|}
\hline \multirow[t]{2}{*}{$\mathrm{SIG}, \mathrm{SI} / \mathrm{mm}$} & \multicolumn{3}{|c|}{ Ischemic stroke of LAA } \\
\hline & OR & $95 \% \mathrm{CI}$ & $p$ value \\
\hline \multicolumn{4}{|l|}{ CCA } \\
\hline \multicolumn{4}{|c|}{ Controls versus all patients } \\
\hline Right & 0.61 & $0.38-0.98$ & 0.041 \\
\hline Left & 0.54 & $0.34-0.85$ & 0.008 \\
\hline \multicolumn{4}{|c|}{ Control versus right-sided stroke of LAA } \\
\hline Right & 0.46 & $0.26-0.80$ & 0.006 \\
\hline Left & 0.81 & $0.56-1.25$ & 0.235 \\
\hline \multicolumn{4}{|c|}{ Control versus left-sided stroke of LAA } \\
\hline Right & 0.87 & $0.78-1.56$ & 0.314 \\
\hline Left & 0.65 & $0.36-1.16$ & 0.142 \\
\hline \multicolumn{4}{|c|}{ ICA } \\
\hline \multicolumn{4}{|c|}{ Controls versus all patients } \\
\hline Right & 0.86 & $0.54-1.38$ & 0.542 \\
\hline Left & 0.61 & $0.38-0.98$ & 0.041 \\
\hline \multicolumn{4}{|c|}{ Control versus right-sided stroke of LAA } \\
\hline Right & 0.50 & $0.29-0.86$ & 0.013 \\
\hline Left & 0.76 & $0.45-1.29$ & 0.307 \\
\hline \multicolumn{4}{|c|}{ Control versus left-sided stroke of LAA } \\
\hline Right & 0.96 & $0.54-1.72$ & 0.898 \\
\hline Left & 0.43 & $0.20-0.94$ & 0.035 \\
\hline
\end{tabular}

Adjusted for age, sex, hypertension, type 2 diabetes, smoking, HDL cholesterol, serum albumin, and ICA stenosis. ICA, internal carotid artery; CCA, common carotid artery; OR, odds ratio; CI, confidence interval; LAA, large artery atherosclerosis; SIG, signal intensity gradient.

\section{Discussion}

The present study showed significant and independent associations between ischemic stroke of LAA and carotid artery ESS, even after adjusting for the potential confounders. The carotid artery ESS was determined with the SIG from TOF-MRA. In both right and left CCAs, the SIG values showed significant associations with the large artery ischemic stroke, whereas in the ICAs, the associations between the SIG and ischemic stroke of LAA were modified by stroke-sidedness, as shown in Table 3. For the right-sided ischemic stroke of LAA, the SIG values from the right ICA showed independent associations, but the left ICA did not, and vice versa. In brief, the presence of lower ESS in both ICA and CCA than controls was associated with the same-sided large artery ischemic stroke.

The CCA and ICA are aligned straightforward but have different hemodynamic features; the CCA represents systemic arterial hemodynamics with a high resistance bed, but ICA represents intracranial hemodynam- ics with a relatively low resistance vascular bed. The CCA, a measurement-site of intima-media thickness, is known to be a representative of arterial status for vascular diseases including coronary artery disease [17]. For the ICA, geometric features including angle, complexity, and flow separation have been reported to be associated with plaque formation and ischemic stroke $[18,19]$. The CCA and ICA are aligned linearly and divided by the bulb portion. Atherosclerotic plaque is known to develop usually in the low ESS zone, such as the outer wall of carotid bulb, and to progress distally (toward the ICA) because of the vicious interaction between blood flow and plaque surface $[20,21]$.

The LAA is a stroke subtype with a large proportion in the TOAST classification, although it varies by age and ethnicity. In patients with ischemic stroke involving unilateral ICA territory, it is important to determine whether or not cervical ICA on the same side is a direct cause [22]. Up to now, the ischemic stroke of LAA subtype is usually diagnosed by the distribution pattern of both ischemic lesion and severe stenosis in a relevant artery including the extracranial carotid artery [4]. However, the severity of extracranial carotid artery stenosis and the incidence of ischemic stroke are not consistent. Even if arterial stenosis is mild, it could be a direct cause of ischemic stroke [23]. In the present study, the prevalence of carotid artery stenosis ( $>50 \%$ stenosis, but not occlusion) was not different between the patients and controls, as shown in Table 1. In addition to arterial stenosis, the pathophysiologic roles of hemodynamic markers such as ESS on ischemic stroke should be studied further to enhance the diagnostic and therapeutic strategies.

The atherosclerotic plaque with a potential lesion of rupture and/or thromboembolism, has been reported to show characteristic pathologic findings, such as thin fibrous cap or intraplaque hemorrhage [24]. However, such features are difficult to find and diagnose. Chemical or imaging biomarkers would be helpful, which could reflect the pathologically compromised lesion and its associated hemodynamics. For example, normal force (pressure) based fractional flow reserve [25] or tangential force based ESS is currently used as the arterial biomarker of hemodynamics in clinical and research fields. In the thrombogenic processes, pathologic ranges of high [26] or low $[27,28]$ arterial ESS have been reported to have direct mechanical, neurohormonal, and even genetic effects on the artery. Abnormal ranges of artery ESS are one of the major causes of cardiac and cerebrovascular events $[29,30]$. 
In the previous study, the present authors reported the significant associations between carotid artery ESS and ischemic stroke, including LAA and SVO [15, 31]. For an ultrasonographic measurement of carotid artery ESS in the study, the subjects with normal or mild carotid stenosis $(<50 \%)$ were enrolled. In addition, those with moderate or severe stenosis $(>50 \%)$ were excluded because of the difficulty in the measurement of diameter along the moderate or severely stenosed and/or calcified segments. In the present study, we enrolled the subjects with varying degrees of carotid stenosis and measured endothelial SIG from all the participants. The endothelial SIG reflects local flow hemodynamics predicted by the Navier-Stokes equation and is not dependent on a representative lumen diameter or mean flow velocity.

Several methods for measuring artery ESS have been proposed, which include computational simulation, such as computational fluid dynamics using the conservation equations of mass and momentum [32], or ultrasonographic methods with the Poiseuille's equation which assumes artery as a long cylindrical structure with a rigid wall [33]. Four-dimensional flow-sensitive MRI can also be used to measure ESS, a method which is especially effective in a large artery such as aorta [34]. In addition to the phase-contrast MR, TOF-MRA also has the potential to determine ESS, as the authors reported previously [11]. The PC MR is based on the Lagrangian approach using the particle tracing, while the SIG is dependent on the Eulerian approach using the discrete signal intensity.

The present study was subject to several limitations. First, it was a case-control study. A prospective cohort study is needed to confirm the reliability of the study results. Second, this study measured carotid artery wall SIG after the incident stroke, and therefore, there is a possibility that the blood flow pattern of the carotid artery might have been altered after the stroke. Third, we measured endothelial SIG by selecting 6 slices around the carotid bifurcation. This method does not include the SIG of the unselected region, so there can be a possibility of selection error. Last, we could not control the anatomical varia- tions of carotid artery which was reported to have an age effect [35], although we excluded the participants aged $<60$ years to minimize the variations.

\section{Conclusions}

ICA endothelial SIG (as a marker of ESS) showed significant and independent associations with the same-sided unilateral ischemic stroke of LAA, whereas CCA endothelial SIG showed a significant association with the presence of large artery ischemic stroke. Although CCA and ICA are aligned linearly, the hemodynamics and their roles in large artery ischemic stroke need to be considered separately.

\section{Statement of Ethics}

This study was conducted following approval from the Ethics Review Committee (CUH2013-03-007-001) of Jeonbuk National University Hospital, and written informed consent was obtained from the participants.

\section{Conflict of Interest Statement}

The authors declare have no conflicts of interest to disclose.

\section{Funding Sources}

Funded by Biomedical Research Institute, Jeonbuk National University Hospital (grant numbers CUH2014-05019002).

\section{Author Contributions}

C.H. analyzed the data and drafted the manuscript. S.H. participated in data analysis and reviewed the drafts and pointed out the problems of the study. Y.C. participated in the study design and reviewed the manuscript. S.K. planned the study, presented the direction to the conclusion, and finally checked the manuscript.

\section{References}

Carotid Endothelial SIG on Large Artery Ischemic Stroke
1 Malhotra K, Gornbein J, Saver JL. Ischemic strokes due to large-vessel occlusions contribute disproportionately to stroke-related dependence and death: a review. Front Neurol. 2017;8:651.

2 Flaherty ML, Kissela B, Khoury JC, Alwell K, Moomaw CJ, Woo D, et al. Carotid artery stenosis as a cause of stroke. Neuroepidemiology. 2013;40(1):36-41.
3 Carallo C, Lucca LF, Ciamei M, Tucci S, de Franceschi MS. Wall shear stress is lower in the carotid artery responsible for a unilateral ischemic stroke. Atherosclerosis. 2006;185(1):108-13.

4 Adams HP Jr, Bendixen BH, Kappelle LJ, Biller J, Love BB, Gordon DL, et al. Classification of subtype of acute ischemic stroke. Definitions for use in a multicenter clinical trial. TOAST. Trial of Org 10172 in acute stroke treatment. Stroke. 1993;24(1):35-41. 
5 Cecchi E, Giglioli C, Valente S, Lazzeri C, Gensini GF, Abbate R, et al. Role of hemodynamic shear stress in cardiovascular disease. Atherosclerosis. 2011;214(2):249-56.

6 Gijsen F, van der Giessen A, van der Steen A, Wentzel J. Shear stress and advanced atherosclerosis in human coronary arteries. J Biomech. 2013;46(2):240-7.

7 Samady H, Eshtehardi P, McDaniel MC, Suo J, Dhawan SS, Maynard C, et al. Coronary artery wall shear stress is associated with progression and transformation of atherosclerotic plaque and arterial remodeling in patients with coronary artery disease. Circulation. 2011;124(7):779-88.

8 Hong JM, Chung CS, Bang OY, Yong SW, Joo IS, Huh K. Vertebral artery dominance contributes to basilar artery curvature and perivertebrobasilar junctional infarcts. J Neurol Neurosurg Psychiatry. 2009;80(10):1087-92.

9 Liu Z, Zhao Y, Wang X, Zhang H, Cui Y, Diao $Y$, et al. Low carotid artery wall shear stress is independently associated with brain whitematter hyperintensities and cognitive impairment in older patients. Atherosclerosis. 2016; 247:78-86.

10 Cebral JR, Yim PJ, Löhner R, Soto O, Choyke PL. Blood flow modeling in carotid arteries with computational fluid dynamics and MR imaging. Acad Radiol. 2002;9(11):1286-99.

11 Han KS, Lee SH, Ryu HU, Park SH, Chung $\mathrm{GH}, \mathrm{Cho}$ YI, et al. Direct assessment of wall shear stress by signal intensity gradient from time-of-flight magnetic resonance angiography. Biomed Res Int. 2017;2017:7087086.

12 Hoogeveen RM, Bakker CJ, Viergever MA Limits to the accuracy of vessel diameter measurement in MR angiography. J Magn Reson Imaging. 1998;8(6): 1228-35.

13 Leng X, Wong KS, Soo Y, Leung T, Zou X, Wang $Y$, et al. Magnetic resonance angiography signal intensity as a marker of hemodynamic impairment in intracranial arterial stenosis. PLoS One. 2013;8(11):e80124.

14 Lee W-J, Jeong S-K, Han K-S, Lee SH, Ryu YJ, Sohn $\mathrm{C}-\mathrm{H}$, et al. Impact of endothelial shear stress on the bilateral progression of unilateral moyamoya disease. Stroke. 2020;51(3): $775-83$.

15 Jeong SK, Lee JY, Rosenson RS. Association between ischemic stroke and vascular shear stress in the carotid artery. J Clin Neurol. 2014;10(2):133-9.
16 Grant EG, Benson CB, Moneta GL, Alexandrov AV, Baker JD, Bluth EI, et al. Carotid artery stenosis: gray-scale and Doppler US diagnosis-society of radiologists in ultrasound consensus conference. Radiology. 2003; 229(2):340-6.

17 Willeit P, Tschiderer L, Allara E, Reuber K, Seekircher L, Gao L, et al. Carotid intima-media thickness progression as surrogate marker for cardiovascular risk: meta-analysis of 119 clinical trials involving 100667 patients. Circulation. 2020;142(7):621-42.

18 Sitzer M, Puac D, Buehler A, Steckel DA, von Kegler S, Markus HS, et al. Internal carotid artery angle of origin: a novel risk factor for early carotid atherosclerosis. Stroke. 2003; 34(4):950-5.

19 Cui Y, Lv X, Wang F, Kong J, Zhao H, Ye Z, et al. Geometry of the carotid artery and its association with pathologic changes in a Chinese population. Front Physiol. 2019;10:1628.

20 Dirksen MT, van der Wal AC, van den Berg FM, van der Loos CM, Becker AE. Distribution of inflammatory cells in atherosclerotic plaques relates to the direction of flow. Circulation. 1998;98(19):2000-3.

21 Olgac U, Poulikakos D, Saur SC, Alkadhi H, Kurtcuoglu V. Patient-specific three-dimensional simulation of LDL accumulation in a human left coronary artery in its healthy and atherosclerotic states. Am J Physiol Heart Circ Physiol. 2009;296(6):H1969-82.

22 Autret A, Pourcelot L, Saudeau D, Marchal C, Bertrand P, de Boisvilliers S. Stroke risk in patients with carotid stenosis. Lancet. 1987; 1(8538):888-90.

23 Zhao H, Zhao X, Liu X, Cao Y, Hippe DS, Sun $\mathrm{J}$, et al. Association of carotid atherosclerotic plaque features with acute ischemic stroke: a magnetic resonance imaging study. Eur J Radiol. 2013;82(9):e465-70.

24 Yuan C, Zhang SX, Polissar NL, Echelard D, Ortiz G, Davis JW, et al. Identification of fibrous cap rupture with magnetic resonance imaging is highly associated with recent transient ischemic attack or stroke. Circulation. 2002;105(2):181-5.

25 Ciccarelli G, Barbato E, Toth GG, Gahl B, Xaplanteris $\mathrm{P}$, Fournier S, et al. Angiography versus hemodynamics to predict the natural history of coronary stenoses: fractional flow reserve versus angiography in multivessel evaluation 2 substudy. Circulation. 2018; 137(14):1475-85.
26 Casa LD, Deaton DH, Ku DN. Role of high shear rate in thrombosis. J Vasc Surg. 2015; 61(4):1068-80.

27 Nesbitt WS, Westein E, Tovar-Lopez FJ, Tolouei E, Mitchell A, Fu J, et al. A shear gradient-dependent platelet aggregation mechanism drives thrombus formation. Nat Med. 2009;15(6):665-73

28 Gimbrone MA Jr, García-Cardeña G. Endothelial cell dysfunction and the pathobiology of atherosclerosis. Circ Res. 2016;118(4):62036.

29 van Ooij P, Markl M, Collins JD, Carr JC, Rigsby C, Bonow RO, et al. Aortic Valve Stenosis Alters Expression Of Regional Aortic Wall Shear Stress: New Insights From A 4-Dimensional Flow Magnetic Resonance Imaging Study Of 571 Subjects. J Am Heart Assoc. 2017;6(9):e005959.

30 Leng X, Lan L, Ip HL, Abrigo J, Scalzo F, Liu $\mathrm{H}$, et al. Hemodynamics and stroke risk in intracranial atherosclerotic disease. Ann Neurol. 2019;85(5):752-64.

31 Jeong SK, Rosenson RS. Shear rate specific blood viscosity and shear stress of carotid artery duplex ultrasonography in patients with lacunar infarction. BMC Neurol. 2013;13(1): 36.

32 Giannopoulos AA, Chatzizisis YS, Maurovich-Horvat P, Antoniadis AP, Hoffmann U, Steigner ML, et al. Quantifying the effect of side branches in endothelial shear stress estimates. Atherosclerosis. 2016;251:213-8.

33 Jiang Y, Kohara K, Hiwada K. Association between risk factors for atherosclerosis and mechanical forces in carotid artery. Stroke. 2000; 31(10):2319-24.

34 van Ooij P, Potters WV, Nederveen AJ, Allen $\mathrm{BD}$, Collins J, Carr J, et al. A methodology to detect abnormal relative wall shear stress on the full surface of the thoracic aorta using four-dimensional flow MRI. Magn Reson Med. 2015;73(3):1216-27.

35 Thomas JB, Antiga L, Che SL, Milner JS, Steinman DA, Spence JD, et al. Variation in the carotid bifurcation geometry of young versus older adults: implications for geometric risk of atherosclerosis. Stroke. 2005;36(11): 2450-6. 